\title{
Optimal Size-Performance Tradeoffs: Weighing PoS Tagger Models
}

\author{
Magnus Jacobsen* \\ Mikkel H. Sørensen* \\ Leon Derczynski \\ IT University of Copenhagen IT University of Copenhagen IT University of Copenhagen \\ mjaceitu.dk \\ mhsoditu.dk \\ leodeitu.dk
}

\begin{abstract}
Improvement in machine learning-based NLP performance are often presented with bigger models and more complex code. This presents a trade-off: better scores come at the cost of larger tools; bigger models tend to require more during training and inference time. We present multiple methods for measuring the size of a model, and for comparing this with the model's performance.

In a case study over part-of-speech tagging, we then apply these techniques to taggers for eight languages and present a novel analysis identifying which taggers are size-performance optimal. Results indicate that some classical taggers place on the size-performance skyline across languages. Further, although the deep models have highest performance for multiple scores, it is often not the most complex of these that reach peak performance.
\end{abstract}

\section{Introduction}

State of the art machine learning has moved from shallow to deep and more complex models. The upwards trend of model complexity has brought with it an increase in hardware demands, including more memory, disk space, and computational power. The full instance of OpenAI's deep learning language model GPT-3, trained on a compressed dataset of 570GB, boasts 175 billion model parameters (Brown et al., 2020); Switch-C, a trillion (Fedus et al., 2021).

However, not all applications of machine learning are on hardware capable of running these models. Devices such as smartphones, hearing aids, or smart speakers are too constrained. Natural Language Processing (NLP) models are important for the devices mentioned above, from voice assistants on smart phones and speakers, to going beyond sound amplification in hearing aids. Using NLP in this context requires considering model size, and

\footnotetext{
* Joint first authors
}

balancing the tradeoff between size and task accuracy. And indeed, larger language models can bring their own drawbacks (Bender et al., 2021).

Determining model size requires metrics. Measuring model filesize in bytes is flawed: some models are compressed; some are uncompressed; some are compressed badly; others embed important functionality in their code instead (Mahoney, 2003); others still impose much larger space constraints at inference time than their dormant, ondisk size indicates. Different starting points also offer varying model size - a custom image might only need a script of a few lines to perform a complex task, that would have many more dependencies on a standard operating system installation. To compare these, common metrics and definitions are required that address these problems.

Our goal is to critically examine the relationship between size complexity and prediction performance of NLP models. We do not examine the time required for training or for inference, only the size of models. We take part-of-speech (PoS) tagging as a case study. PoS tagging is a well-defined problem, with well-defined sets of inputs and labeled outputs. Readily measurable output benefits performance measurements and comparisons. PoS tagging is also no different from the trend in machine learning, where better has become coupled to bigger.

Our research questions are: RQ1 How can we measure the size of an NLP tool in a reproducible, comparable way? RQ2 How can we determine which tools are size-performance optimal?

In relation to prediction performance, we probe how well taggers handle a diverse range of languages. This will test their robustness and ability to generalise when presented with different inputs. In relation to size we intend to investigate models of varying complexity and parameter count. This will show how PoS taggers of different size perform. We explore various PoS tagging approaches 
and methods for minimizing the size of machine learning models. Further, we evaluate the prediction accuracy of various PoS taggers in comparison to their size, and finally discuss these findings in relation to further research to model size optimality.

\section{Related Work}

As machine learning models have grown, more research has investigated optimizing model size.

Compression has shown that large networks rarely make full use of the space they take up (Han et al., 2016). One form of model compression is to use limit precision for parameters. Gupta et al. (2015) experimented with limited precision with fixed-point numbers. Similarly, Wang et al. (2018) experimented with quantized limited precision floating point numbers. Both compression schemes needed stochastic rounding to preserve accuracy. The extreme cases of limited precision are binary or ternary networks, where weights are either -10 or 1 . This was explored for LSTM networks (Ardakani et al., 2019) and CNNs (Ignatov and Ignatov, 2020).

The advent of pre-trained embeddings and transformer models, such as BERT (Devlin et al., 2019), prompted research into minimizing their size. Word embeddings are an integral part in deep learning PoS taggers, but large. Several works have explored embedding compression (Chen et al., 2016; Tissier et al., 2019; Kim et al., 2020; MelasKyriazi et al., 2020; Liao et al., 2020).

Another form of resource-constrained NLP is with low-resource languages or datasets. Research into unsupervised PoS tagging on low resource languages has been done by Buys and Botha (2016); Cardenas et al. (2019). Ezen-Can (2020) evaluated the performance of BERT on a small dataset.

The most similar research to ours is Szymanski and Gorman (2020), who use a Bayesian approach to evaluate six PoS taggers across two datasets. However, they did not conduct size measurements or analysis, and only covered English.

\section{Method}

We evaluated ten PoS taggers across eight languages, to see the size vs. accuracy trade-off of classical and contemporary taggers, measuring performance using two accuracy metrics and three size metrics.

\subsection{Taggers}

We reviewed and experimented on five taggers that utilize classical non-neural machine learning techniques. We did the same for five modern taggers utilizing deep learning. The majority of these taggers achieved state-of-the-art performance at the time of their inception.

We modified the code for some taggers, to make them compatible with our testing platform. We also changed how some of them parsed input to make them compatible with testing data. For some taggers, we created an inference function, as it was missing. We did not alter the model architecture in any way for any of the taggers.

\subsubsection{Classical Taggers}

Brill Tagger Rule-based tagger (Brill, 1992). Achieved a state-of-the-art accuracy for its time of $95 \%$ on the English Brown Corpus.

Trigrams'n'Tags (TnT) Hidden Markov Model (HMM) (Brants, 2000). Achieved a state-of-the-art accuracy for its time of $96.7 \%$ on the English WSJ PTB (Marcus et al., 1993).

Stanford Tagger Uses maximum-entropy approximation and a cyclic dependency network (Toutanova et al., 2003). Scored state-ofthe-art accuracy at the time: $97.24 \%$ on PTB.

SVMTool Utilizes Support Vector Machines and achieved an accuracy of $97.16 \%$ on the Penn Treebank corpus (Giménez and Màrquez, 2004). We use the version written in Perl.

HMM (NLTK) First-order HMM from the Natural Language Toolkit (NLTK) (Bird et al., 2002).

\subsubsection{Deep Learning Taggers}

All of the modern taggers we investigated utilized recurrent neural network (RNN) architectures. Specifically, LSTM networks are utilized by all of them to boost state-of-the-art accuracy. All the taggers also employ either word or subword embeddings, or a combination of these. Embeddings and LSTM networks contain many parameters and therefore require more memory and disk space to use than classical approaches.

BiLSTM (Plank) Uses an auxiliary loss function to handle rare words (Plank et al., 2016). Reached state-of-the-art scores for its time on 22 languages. 
BiLSTM (Yasunaga) Employs adversarial training for regularization and a conditional random field (CRF) for prediction (Yasunaga et al., 2018). Achieved competitive results across different languages, including an accuracy of $97.58 \%$ on PTB.

Flair Uses a novel embedding form, "contextual string embeddings" (Akbik et al., 2018). Achieved a state-of-the-art accuracy for its time of $97.82 \%$.

Meta-BiLSTM Combines three separately trained BiLSTM neural networks (Bohnet et al., 2018). Achieved state-of-the-art accuracy for its time of $97.96 \%$ on the PTB corpus.

BiLSTM (Heinzerling) Uses a combination of sub-word embeddings, including the BERT transformer model (Heinzerling and Strube, 2020). Gave competitive accuracy across 27 languages.

\subsection{Metrics}

Measuring the size and accuracy trade-off of machine learning models is not a trivial issue. For different applications, different size constraints might apply. To get a broad view of performance, we measured each tagger with respect to three size metrics and two accuracy metrics. We measured token accuracy and sentence accuracy. Below, we outline the three size metrics, and how we measure them.

Memory We measured memory usage during inference on test data. For each tagger, we ran inference in an isolated process and polled the memory usage twice every second. The reported memory usage for a tagger is the average usage across these measurements. This should provide a realistic view on how much memory a tagger would consume in a typical application.

Model Size (Uncompressed) We measured the disk space required by a tagger by how many bytes its trained parameters took up on disk. We also included any pre-trained embeddings for the tagger in this measurement. These were included because embeddings can be seen as additional trained parameters for the model.

Model Size (Compressed) We compressed necessary model files for a tagger to the lossless .xz archiving format. This is not to be confused with model compression as mentioned in section 2 . This gives a naive lower bound for the potential size of a trained model, without altering its architecture.

\subsection{Datasets}

To test the multilingual capabilities of the taggers, we chose a diverse selection of languages. These languages span different families and contain different morphological characteristics. The languages used are in table 1 .

\begin{tabular}{l|ccc}
\hline & Code & Family & Branch \\
\hline Arabic & ar & Afro-Asiatic & Semitic \\
Chinese & zh & Sino-Tibetan & Sinitic \\
Danish & da & Indo-European & Germanic \\
English & en & Indo-European & Germanic \\
Hindi & hi & Indo-European & Indo-Iranian \\
Russian & ru & Indo-European & Balto-Slavic \\
Spanish & es & Indo-European & Italic \\
Turkish & tr & Turkic & Common Turkic \\
\hline
\end{tabular}

Table 1: Grouping of languages. Codes are ISO 639-1.

We used Universal Dependencies (UD) 2.6 datasets (Nivre et al., 2020). UD provides uniformly labelled datasets for a large array of languages. The languages together with the chosen dataset for each, are in table 2. The Chinese dataset uses Traditional Chinese characters. The split were pre-determined by the UD datasets.

\begin{tabular}{l|cccc}
\hline & Dataset & Tokens & Sentences & Splits \\
\hline Arabic & PADT & $242 \mathrm{~K}$ & 7,664 & $80 / 10 / 10$ \\
Chinese & GSD & $123 \mathrm{~K}$ & 4,997 & $80 / 10 / 10$ \\
Danish & DDT & $101 \mathrm{~K}$ & 5,512 & $80 / 10 / 10$ \\
English & GUM & $113 \mathrm{~K}$ & 5,961 & $72 / 14 / 14$ \\
Hindi & HDTB & $352 \mathrm{~K}$ & 16,647 & $80 / 10 / 10$ \\
Russian & GSD & $98 \mathrm{~K}$ & 5,030 & $76 / 12 / 12$ \\
Spanish & AnCora & $548 \mathrm{~K}$ & 17,680 & $80 / 10 / 10$ \\
Turkish & IMST & $56.4 \mathrm{~K}$ & 5,635 & $66 / 17 / 17$ \\
\hline
\end{tabular}

Table 2: Datasets used for experiments. Splits are approximate percentages of total tokens for training, development, and testing sets, respectively.

Data Curation Some taggers expected input data structured differently from the files found in UD. To deal with this issue, we created versions of the datasets where sentences were merely token and tag pairs. We also removed non-tagged multitoken words from the original .conllu files. None of the taggers expected multitoken words. These were introduced with version 2 of UD.

For SVMTool we made some minor data modifications. We had to guarantee that every sentence ended with a full stop. 
Pre-Trained Embeddings Some taggers used embeddings, often trained unsupervised over a large corpus. They encapsulate contextual word information, represented as fixed-size vectors.

Polyglot word embeddings (Al-Rfou et al., 2013) with a dimensionality of 64 were used for BiLSTM (Plank), BiLSTM (Yasunaga), and Meta-BiLSTM. BPEmb byte-pair embeddings (Heinzerling and Strube, 2019) with 100 dimensions were used for the BERT-BPEmb tagger. A combination of Polyglot and FastText embeddings (Joulin et al., 2017) was used with Flair, the latter having 300 dimensions.

\subsection{Experiment Setup}

To conduct experiments with taggers written in different programming languages, we needed a flexible testing platform. We built this platform using Python.

The specifications for the hardware used for experiments are: dual Intel Xeon Platinum 8176z CPU; 503GB memory; Ubuntu 18.04 (64-bit); Python 3.8.5.

\section{Results}

Here we report measurement results from our experiments, for both accuracy and size metrics. We then link these metrics to investigate tradeoffs.

\subsection{Accuracy Measurements}

The accuracy measurements from our experiments are found in table 3 and 4 . The first table shows results when predicting individual tags for tokens. The second table shows accuracy for predicting complete sentences. All measurements were done from taggers predicting on unseen test datasets.

Token Accuracy Results for token accuracy are in Table 4. Deep learning models performed better almost across the board. The network that performed best on average, across all languages, was Flair. These results are not surprising. Flair uses pre-trained embeddings with more dimensions than the other deep learning taggers.

Flair performed best on seven individual languages. The only language it performed poorly on was Chinese. It had the lowest accuracy among the deep learning models. Chinese is the language with the 3rd lowest average accuracy among all taggers. Even still, Flair's low performance is remarkable. This could be because its architecture is built around splitting words and tokens into sequences of characters (Akbik et al., 2018). Chinese is a logographic languages, which makes this difficult. BERT-BPEmb performs better than all other taggers on Chinese. This suggests that the attention architecture found in its BERT component (Devlin et al., 2019) is better than BiLSTMs at learning Chinese language form.

Among classical taggers, both SVMTool and the Stanford Tagger perform well. They are behind all the deep learning models in performance, but lie closer to these than to the other classical taggers. Figure 1 shows how competitive the accuracy of SVMTool is in comparison to the deep learning taggers. The gap in performance for Brill, HMM, and $\mathrm{TnT}$ is considerably larger.

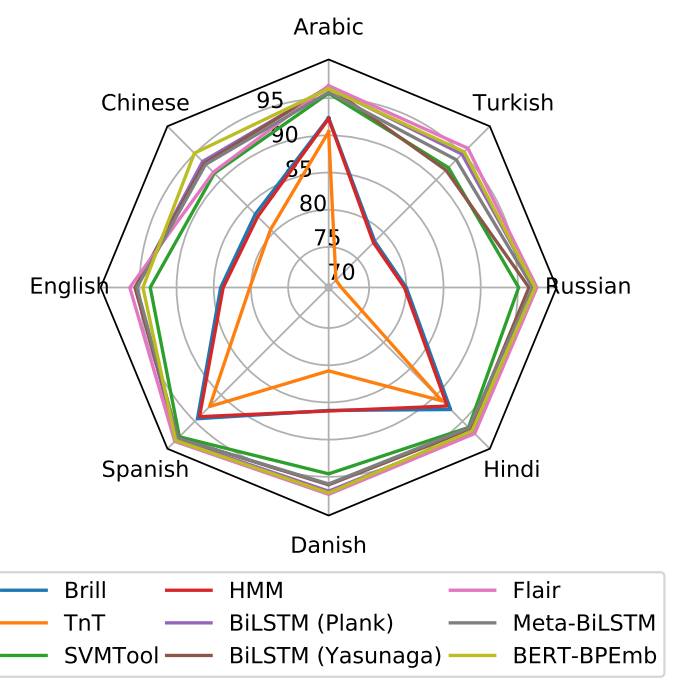

Figure 1: Taggers token performance for each language. Stanford Tagger excluded.

Sentence Accuracy Results for sentence accuracy are in table 4 . Here, the difference between classical and deep learning taggers is even more pronounced. The performance of the Stanford Tagger and SVMTool are far better than the other classical taggers. Again, the deep learning taggers perform better across the board. Flair has the best performance on the same seven languages as with token accuracy. It also has the highest average accuracy across all languages. As with token accuracy, BERT-BPEmb has best performance on Chinese.

Sentence accuracy provides another perspective on how versatile and robust a tagger is. If a given tagger performs well in token accuracy, but poorly in sentence accuracy, it might not grasp the context of words and tags in sentences. In that case, it has 


\begin{tabular}{l|cccccccccc|c}
\hline & Brill & TnT & SVMTool & $\begin{array}{c}\text { Stanford } \\
\text { Tagger }\end{array}$ & HMM & $\begin{array}{c}\text { BiLSTM/ } \\
\text { Plank }\end{array}$ & $\begin{array}{c}\text { BiLSTM/ } \\
\text { Yasunaga }\end{array}$ & Flair & $\begin{array}{c}\text { Meta- } \\
\text { BiLSTM }\end{array}$ & $\begin{array}{c}\text { BERT- } \\
\text { BPEmb }\end{array}$ & Avg. \\
\hline ar & 92.36 & 90.49 & 95.66 & 95.73 & 92.25 & 96.43 & 96.55 & $\mathbf{9 6 . 6 8}$ & 95.80 & 96.27 & 94.82 \\
zh & 83.50 & 80.59 & 91.28 & 91.84 & 83.04 & 93.51 & 93.17 & 91.47 & 92.95 & $\mathbf{9 5 . 0 8}$ & 89.64 \\
en & 84.07 & 80.15 & 93.54 & 93.64 & 83.73 & 95.50 & 95.60 & $\mathbf{9 6 . 2 9}$ & 95.34 & 94.52 & 91.24 \\
es & 94.50 & 92.18 & 97.93 & 98.12 & 94.11 & 98.50 & 98.19 & $\mathbf{9 8 . 8 1}$ & 98.22 & 98.64 & 96.92 \\
da & 86.08 & 80.76 & 94.60 & - & 86.15 & 96.93 & 96.08 & $\mathbf{9 7 . 3 6}$ & 95.96 & 97.17 & 83.11 \\
hi & 92.75 & 91.10 & 96.16 & - & 92.09 & 97.10 & 96.50 & $\mathbf{9 7 . 3 2}$ & 96.23 & 96.82 & 85.61 \\
ru & 80.00 & 71.26 & 95.10 & - & 79.74 & 97.36 & 96.49 & $\mathbf{9 7 . 5 5}$ & 97.00 & 97.30 & 81.18 \\
tr & 78.33 & 70.95 & 92.33 & - & 78.08 & 94.90 & 91.86 & $\mathbf{9 6 . 0 2}$ & 93.83 & 95.34 & 79.16 \\
\hline Avg. & 86.45 & 82.19 & 94.58 & - & 86.15 & 96.28 & 95.55 & $\mathbf{9 6 . 4 4}$ & 95.67 & 96.39 & - \\
\hline
\end{tabular}

Table 3: Token accuracy on test set, for all taggers on all supported languages. Numbers are in percent. Bold is best row score.

\begin{tabular}{l|cccccccccc|c}
\hline & Brill & TnT & SVMTool & $\begin{array}{c}\text { Stanford } \\
\text { Tagger }\end{array}$ & HMM & $\begin{array}{c}\text { BiLSTM/ } \\
\text { Plank }\end{array}$ & $\begin{array}{c}\text { BiLSTM/ } \\
\text { Yasunaga }\end{array}$ & Flair & $\begin{array}{c}\text { Meta- } \\
\text { BiLSTM }\end{array}$ & $\begin{array}{c}\text { BERT- } \\
\text { BPEmb }\end{array}$ & Avg. \\
\hline r & 23.68 & 17.50 & 37.79 & 38.53 & 23.24 & 44.26 & 46.00 & $\mathbf{4 6 . 7 6}$ & 37.50 & 44.56 & 35.98 \\
zh & 6.40 & 2.40 & 22.60 & 26.80 & 5.60 & 32.60 & 29.60 & 24.40 & 26.00 & $\mathbf{3 9 . 6 0}$ & 21.60 \\
en & 17.30 & 12.13 & 43.03 & 45.17 & 16.63 & 52.36 & 54.00 & $\mathbf{5 9 . 8 9}$ & 53.15 & 48.31 & 40.20 \\
es & 28.76 & 18.07 & 60.37 & 64.73 & 25.62 & 68.74 & 64.41 & $\mathbf{7 4 . 3 2}$ & 64.44 & 72.57 & 54.20 \\
da & 17.52 & 8.50 & 46.02 & - & 17.52 & 61.06 & 57.40 & $\mathbf{6 8 . 3 2}$ & 55.22 & 65.84 & 39.74 \\
hi & 33.43 & 24.23 & 56.35 & - & 28.98 & 63.12 & 59.13 & $\mathbf{6 7 . 1 6}$ & 54.22 & 62.65 & 44.93 \\
ru & 10.32 & 2.33 & 48.42 & - & 9.32 & 69.05 & 59.33 & $\mathbf{6 9 . 2 2}$ & 63.56 & 64.89 & 39.64 \\
tr & 19.02 & 10.17 & 51.88 & - & 18.62 & 62.46 & 49.33 & $\mathbf{6 9 . 8 9}$ & 57.68 & 65.11 & 40.42 \\
\hline Avg. & 19.55 & 11.92 & 45.81 & - & 18.19 & 56.71 & 52.40 & $\mathbf{6 0 . 0 0}$ & 51.47 & 57.94 & - \\
\hline
\end{tabular}

Table 4: Sentence accuracy on test set, for all taggers on all supported languages. Numbers are in percent.

merely learned what token generally corresponds to which tag.

\subsection{Size Measurements}

Size measurements across the three chosen metrics are in Table 5. These are averaged across results for all languages.

\begin{tabular}{l|ccc}
\hline & Memory & Model & $\begin{array}{c}\text { Model } \\
\text { Compr. }\end{array}$ \\
\hline Brill & $1.04 \mathrm{e} 5$ & $1.99 \mathrm{e} 3$ & $1.78 \mathrm{e} 2$ \\
TnT & $1.99 \mathrm{e} 5$ & $6.85 \mathrm{e} 2$ & $1.32 \mathrm{e} 2$ \\
SVMTool & $5.77 \mathrm{e} 4$ & $4.03 \mathrm{e} 3$ & $7.37 \mathrm{e} 2$ \\
Stanford Tagger & $2.92 \mathrm{e} 5$ & $5.42 \mathrm{e} 3$ & $2.01 \mathrm{e} 3$ \\
HMM & $1.03 \mathrm{e} 5$ & $4.45 \mathrm{e} 2$ & $1.30 \mathrm{e} 2$ \\
BiLSTM/ Plank & $1.18 \mathrm{e} 6$ & $1.46 \mathrm{e} 5$ & $4.87 \mathrm{e} 4$ \\
BiLSTM/ Yasunaga & $5.08 \mathrm{e} 6$ & $4.10 \mathrm{e} 4$ & $3.37 \mathrm{e} 4$ \\
Flair & $5.50 \mathrm{e} 6$ & $7.96 \mathrm{e} 5$ & $4.68 \mathrm{e} 5$ \\
Meta-BiLSTM & $2.74 \mathrm{e} 7$ & $2.88 \mathrm{e} 5$ & $2.60 \mathrm{e} 5$ \\
BERT-BPEmb & $6.80 \mathrm{e} 7$ & $1.46 \mathrm{e} 6$ & $1.33 \mathrm{e} 6$ \\
\hline
\end{tabular}

Table 5: Average size after training, across all languages for each tagger. All sizes are in kilobytes.
Memory SVMTool uses the least memory during inference of all taggers. This is due to its reliance on lightweight SVM techniques. BiLSTM (Plank) is the least memory intensive of the deep learning taggers. This is likely due to its machine learning framework DyNet. This framework is more lightweight than TensorFlow or PyTorch which is used by the three largest models. BiLSTM (Yasunaga) use Theano, which is also relatively lightweight. We did not limit available memory for any of the models. Putting constraints on the available memory for the taggers might result in lower numbers. I.e. these figures should be seen as an upper bound.

Model Size (Uncompressed) Using this metric, the amount of parameters for the classical taggers are magnitudes smaller than for the deep learning models. The smallest amount belongs to the Stanford Tagger. This is an outlier because it contains hard-coded language-specific features in its code base. This enables the small size on disk for the stored parameters. BiLSTM (Yasunaga) is the most lightweight of the deep learning models. Plank is 
larger due to storing its parameters in a less efficient format. It is worth remembering that embeddings are included in model size. This factors into the larger sizes of the deep learning models.

Model Size (Compressed) SVMTool and BiLSTM (Plank) gains the most from lossless xz compression. This is because their trained parameters are stored in plain text. The size rankings compared to the previous metric is otherwise unchanged.

\subsection{Size-Accuracy Efficiency}

The eight plots in figure 2 show memory usage during inference on the $\mathrm{x}$-axis, and token accuracy on the y-axis. The figure includes plots with measurements across all eight languages for all taggers. The Stanford Tagger is included for the languages it supports. The red dotted line in each plot represents the skyline (Börzsönyi et al., 2001). This indicates the measured optimum between memory usage and accuracy. The skyline intersects the optimal taggers. The taggers below the skyline are dominated by better optimums.

Figure 3 and 4 summarize how often each tagger emerges as the most optimal choice for a specific metric. I.e. how often a tagger is on the skyline for a given size and accuracy metric.

With memory usage SVMTool dominates all taggers with lower accuracy, as none of them have a footprint that is smaller. When more memory is available, BiLSTM (Plank) or Flair stands out as the two best choices. Flair reaches highest token accuracy for seven of the eight languages, but requires more memory than the Plank model. Even though BERT-BPEmb has a performance almost at Flair's level, it only appears on the skyline for Chinese. This is because Flair not only has a better performance, but also a smaller footprint.

When it comes to model size and compressed model size, Brill and HMM are not dominated in the same fashion as for memory consumption. HMM is efficient for all languages, but neither reach strong peak accuracy. The HMM model from NLTK performs well in this metric because of its small size. SVMTool is efficient for all languages when it is compressed.

Looking at plots from Figure 3 and 4, SVMTool, BiLSTM (Plank), and Flair are the most generally efficient taggers. They achieve optimal tradeoff in size vs. accuracy across most metrics for the tested taggers. SVMTool is the most versatile choice to use on embedded devices. It keeps a good bal- ance of small size across metrics with decent accuracy. While the remaining classical taggers are more lightweight in certain size metrics, they do not manage the same consistent accuracy as SVMTool.

These results show that a larger and more complex model does not guarantee consistent performance. Flair has the highest consistent accuracy across languages, but is not the largest model for any size metrics. The same is true for SVMTool. It outperforms all other classical taggers except Stanford Tagger in terms of performance. Stanford Tagger is larger than SVMTool and only support a small set of languages.

\section{Discussion}

Among the deep learning taggers, BiLSTM (Plank) and Flair are the ones that are most often efficient, seen in Figures 3 and 4. If one was to compress contemporary PoS taggers for use on constrained devices, these two taggers are obvious candidates. For example, using an aggressive compression pipeline with pruning, quantization, and weight sharing, as done by Han et al. (2016).

All deep learning taggers use some form of embeddings. For instance, most of Flair's size is due to the dimensions of the FastText embeddings. Compression of embeddings could help lower the memory and disk space required by the deep learning taggers. One could examine the approach by Kim et al. (2020), where they learn NLP task-specific features and compress embeddings accordingly. A different approach by Liao et al. (2020) used quantization and dimensionality reduction to optimize the encoding of word embeddings, while preserving relevant information.

\subsection{Limitations}

Code Base Measurement As a fourth size metric, we considered measuring the size of the code base underlying each tagger. Estimating this is not trivial. The taggers from our experiments are written in various high-level languages and use various frameworks. A lot of the actual code logic is abstracted away in dependency chains. This obfuscates the actual size of the code required to run the taggers.

Production code is often rewritten to be more compact, without general purpose frameworks, and in a low-level language. This makes measurement of high-level language code less interesting. 

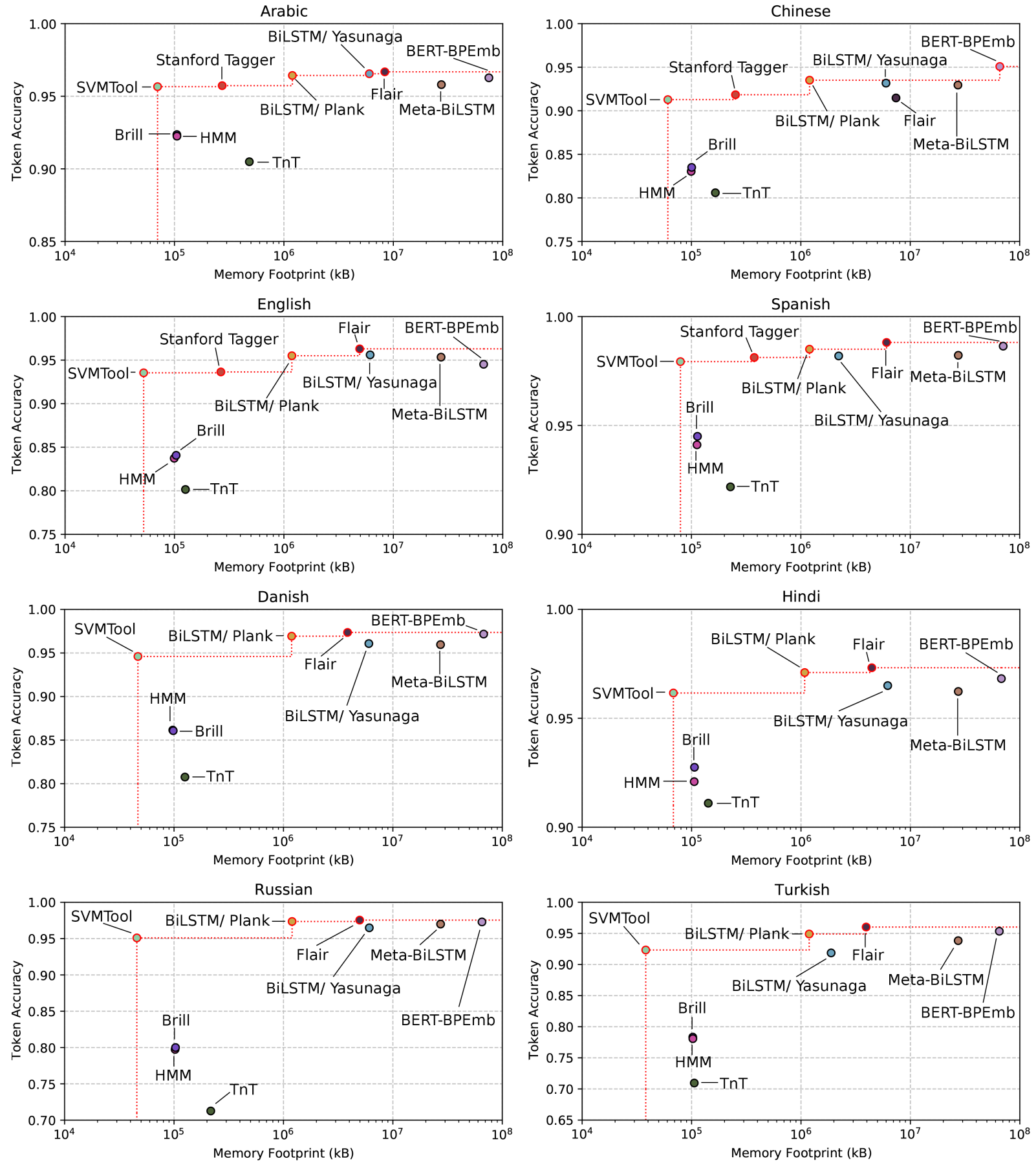

Figure 2: Skyline plots of taggers for all eight languages showing token accuracy vs. memory usage 
Token accuracy
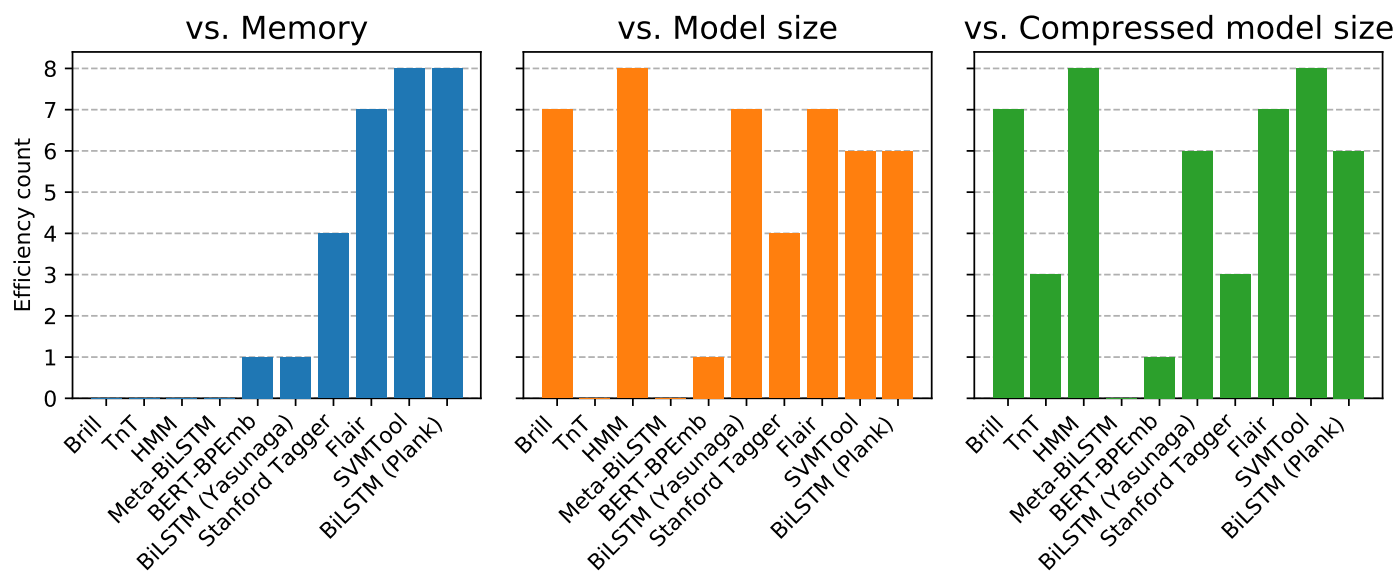

Figure 3: Token accuracy optimality: number of languages for which each tagger was on skyline

\section{Sentence accuracy}
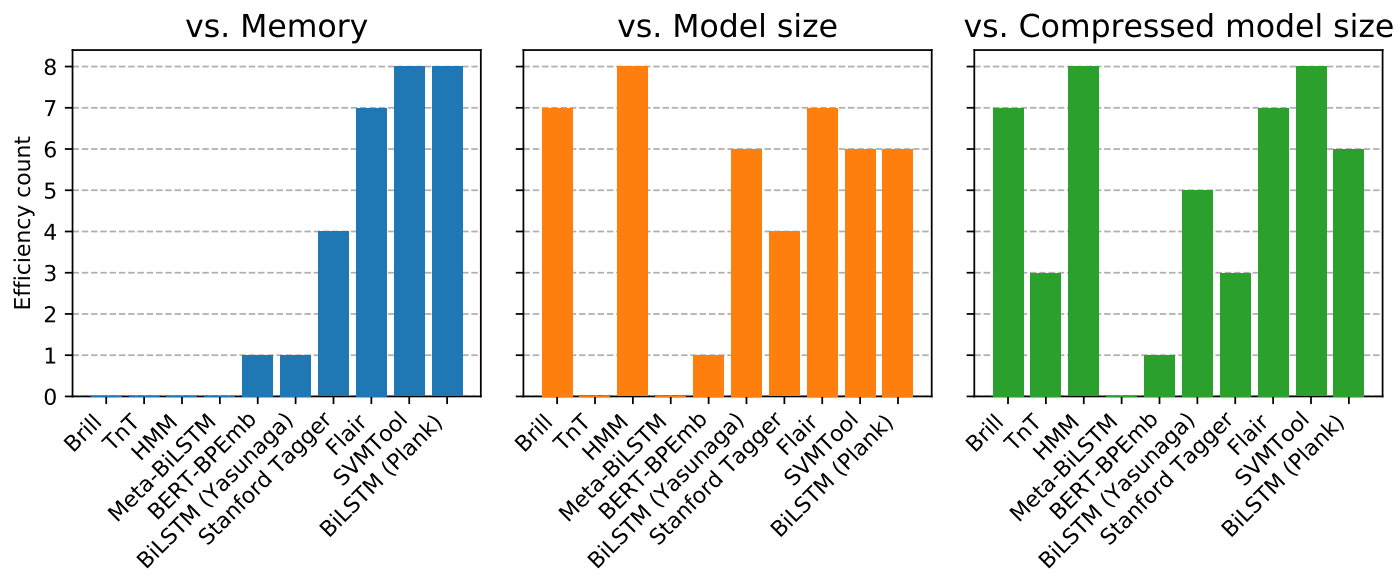

Figure 4: Sentence accuracy optimality: number of languages for which each tagger was on skyline

Visualisation In our experiments we trained ten PoS taggers on eight different languages. We measured two accuracy metrics and four size metrics. Creating plots for every combination of metrics would result in 48 individual plots. This would be confusing and not add value. Merely taking a tagger's average accuracy across all languages would obfuscate interesting information: Languages that have different characteristics are not directly comparable. Additionally, datasets for languages had varying sizes. We limited the featured skyline plots to a combination of token prediction accuracy and memory footprint. Token accuracy is the most common accuracy metric for PoS tagging. Memory usage is interesting because it captures the effective usage of the code base and model parameters at runtime, where memory is often a bottleneck on resource-constrained devices.

\section{Conclusion}

This paper addressed how to measure the size of NLP models and how to select tools that optimise both predictive performance and size. We presented multiple size metrics, and a multi-lingual comparison of a broad range of part-of-speech taggers. We have measured their token and sentence accuracy, along with their size footprints. When combining these two groups of metrics, size and accuracy, we have shown how to determine which taggers are efficient, and which are not.

The methods introduced give means for selecting optimal tool under either size or performance constraints (or both), as well as identifying which tools present no efficiency. Further, the results show that 
learning and encoding general knowledge, from a wide array of languages and datasets, is possible even with a small size footprint.

\section{References}

Alan Akbik, Duncan Blythe, and Roland Vollgraf. 2018. Contextual String Embeddings for Sequence Labeling. Proceedings of the 27th International Conference on Computational Linguistics.

Rami Al-Rfou, Bryan Perozzi, and Steven Skiena. 2013. Polyglot: Distributed word representations for multilingual NLP. In CoNLL 2013 - 17th Conference on Computational Natural Language Learning, Proceedings.

Arash Ardakani, Zhengyun Ji, Sean C. Smithson, Brett H. Meyer, and Warren J. Gross. 2019. Learning recurrent binary/ternary weights. In 7th International Conference on Learning Representations, ICLR 2019.

Emily M Bender, Timnit Gebru, Angelina McMillanMajor, and Shmargaret Shmitchell. 2021. On the dangers of stochastic parrots: Can language models be too big. Proceedings of FAccT.

Steven Bird, Steven Bird, and Edward Loper. 2002. NLTK : The natural language toolkit NLTK : The Natural Language Toolkit. Proceedings of the ACL02 Workshop on Effective tools and methodologies for teaching natural language processing and computational linguistics-Volume 1.

Bernd Bohnet, Ryan McDonald, Gonçalo Simões, Daniel Andor, Emily Pitler, and Joshua Maynez. 2018. Morphosyntactic tagging with a meta-bilSTM model over context sensitive token encodings. In ACL 2018 - 56th Annual Meeting of the Association for Computational Linguistics, Proceedings of the Conference (Long Papers).

S. Börzsönyi, D. Kossmann, and K. Stocker. 2001. The skyline operator. In Proceedings - International Conference on Data Engineering.

Thorsten Brants. 2000. TnT - A Statistical Part-ofSpeech Tagger. CoRR, cs.CL/0003.

Eric Brill. 1992. A simple rule-based part of speech tagger. In ANLC '92: Proceedings of the third conference on Applied natural language processing, page 152 .

Tom B. Brown, Benjamin Mann, Nick Ryder, Melanie Subbiah, Jared Kaplan, Prafulla Dhariwal, Arvind Neelakantan, Pranav Shyam, Girish Sastry, Amanda Askell, Sandhini Agarwal, Ariel Herbert-Voss, Gretchen Krueger, Tom Henighan, Rewon Child, Aditya Ramesh, Daniel M. Ziegler, Jeffrey Wu, Clemens Winter, Christopher Hesse, Mark Chen, Eric Sigler, Mateusz Litwin, Scott Gray, Benjamin Chess, Jack Clark, Christopher Berner, Sam McCandlish, Alec Radford, Ilya Sutskever, and Dario Amodei. 2020. Language models are few-shot learners.

Jan Buys and Jan A. Botha. 2016. Cross-lingual morphological tagging for low-resource languages. In 
54th Annual Meeting of the Association for Computational Linguistics, ACL 2016 - Long Papers.

Ronald Cardenas, Ying Lin, Heng Ji, and Jonathan May. 2019. A grounded unsupervised universal partof-speech tagger for low-resource languages. In NAACL HLT 2019 - 2019 Conference of the North American Chapter of the Association for Computational Linguistics: Human Language Technologies Proceedings of the Conference.

Yunchuan Chen, Lili Mou, Yan $\mathrm{Xu}, \mathrm{Ge} \mathrm{Li}$, and Zhi Jin. 2016. Compressing neural language models by sparse word representations. In 54th Annual Meeting of the Association for Computational Linguistics, ACL 2016 - Long Papers.

Jacob Devlin, Ming Wei Chang, Kenton Lee, and Kristina Toutanova. 2019. BERT: Pre-training of deep bidirectional transformers for language understanding. In NAACL HLT 2019 - 2019 Conference of the North American Chapter of the Association for Computational Linguistics: Human Language Technologies - Proceedings of the Conference.

Aysu Ezen-Can. 2020. A comparison of LSTM and BERT for small corpus.

William Fedus, Barret Zoph, and Noam Shazeer. 2021. Switch transformers: Scaling to trillion parameter models with simple and efficient sparsity. arXiv preprint arXiv:2101.03961.

Jesús Giménez and Lluís Màrquez. 2004. SVMTool: A general POS tagger generator based on support vector machines. In Proceedings of the 4th International Conference on Language Resources and Evaluation, LREC 2004.

Suyog Gupta, Ankur Agrawal, Kailash Gopalakrishnan, and Pritish Narayanan. 2015. Deep learning with limited numerical precision. In 32nd International Conference on Machine Learning, ICML 2015.

Song Han, Huizi Mao, and William J. Dally. 2016 Deep compression: Compressing deep neural networks with pruning, trained quantization and Huffman coding. In 4th International Conference on Learning Representations, ICLR 2016 - Conference Track Proceedings.

Benjamin Heinzerling and Michael Strube. 2019 BPEMB: Tokenization-free pre-trained subword embeddings in 275 languages. In LREC 2018 - 11th International Conference on Language Resources and Evaluation.

Benjamin Heinzerling and Michael Strube. 2020. Sequence tagging with contextual and non-contextual subword representations: A multilingual evaluation. In ACL 2019 - 57th Annual Meeting of the Association for Computational Linguistics, Proceedings of the Conference.
Dmitry Ignatov and Andrey Ignatov. 2020. Controlling information capacity of binary neural network. Pattern Recognition Letters.

Armand Joulin, Edouard Grave, Piotr Bojanowski, and Tomas Mikolov. 2017. Bag of tricks for efficient text classification. In 15th Conference of the European Chapter of the Association for Computational Linguistics, EACL 2017 - Proceedings of Conference.

Yeachan Kim, Kang-Min Kim, and SangKeun Lee. 2020. Adaptive Compression of Word Embeddings. In ACL - 58th Annual Meeting of the Association for Computational Linguistics, Proceedings of the Conference (Long Papers).

Siyu Liao, Jie Chen, Yanzhi Wang, Qinru Qiu, and Bo Yuan. 2020. Embedding compression with isotropic iterative quantization.

Matt Mahoney. 2003. Better archiver with recursive functionality (BARF). http://mattmahoney.net/dc/barf.html.

M. Marcus, B Santorini, and M. Marcinkiewicz. 1993. Building a Large Annotated Corpus of English: The Penn Treebank. Computational linguistics - Association for Computational Linguistics (Print).

Luke Melas-Kyriazi, George Han, and Celine Liang. 2020. Generation-distillation for efficient natural language understanding in low-data settings.

Joakim Nivre, Marie Catherine de Marneffe, Filip Ginter, Jan Haji, Christopher D. Manning, Sampo Pyysalo, Sebastian Schuster, and Francis Tyers Daniel Zeman. 2020. Universal dependencies v2: An evergrowing multilingual treebank collection. In LREC 2020 - 12th International Conference on Language Resources and Evaluation, Conference Proceedings.

Barbara Plank, Anders Søgaard, and Yoav Goldberg. 2016. Multilingual part-of-speech tagging with bidirectional long short-term memory models and auxiliary loss. In 54th Annual Meeting of the Association for Computational Linguistics, ACL 2016 - Short Papers.

Piotr Szymanski and Kyle Gorman. 2020. Is the best better? Bayesian statistical model comparison for natural language processing.

Julien Tissier, Christophe Gravier, and Amaury Habrard. 2019. Near-lossless binarization of word embeddings. In 33rd AAAI Conference on Artificial Intelligence, AAAI 2019, 31st Innovative Applications of Artificial Intelligence Conference, IAAI 2019 and the 9th AAAI Symposium on Educational Advances in Artificial Intelligence, EAAI 2019.

Kristina Toutanova, Dan Klein, Christopher D. Manning, and Yoram Singer. 2003. Feature-rich part-ofspeech tagging with a cyclic dependency network. In In Proceedings of HLT-NAACL 2003, pages 173180. 
Naigang Wang, Jungwook Choi, Daniel Brand, Chia Yu Chen, and Kailash Gopalakrishnan. 2018. Training deep neural networks with 8-bit floating point numbers. In Advances in Neural Information Processing Systems.

Michihiro Yasunaga, Jungo Kasai, and Dragomir Radev. 2018. Robust multilingual part-of-speech tagging via adversarial training. In NAACL HLT 2018 - 2018 Conference of the North American Chapter of the Association for Computational Linguistics: Human Language Technologies - Proceedings of the Conference. 